\title{
Evaluation of the immediate post-operative procedure after dental intervetions. 24 hours follow up study. Epico Study
}

\author{
José V. Bagán ${ }^{1}$, Begoña Soler-López ${ }^{2}$, Épico-Study-Group ${ }^{3}$
}

\author{
${ }^{1}$ MD Head of the Dentistry Department, Hospital General Universitary of Valencia, Avenida Tres Cruces, s/n, 46014 Valencia, \\ Spain \\ ${ }^{2}$ MD Medical Director, E-C-BIO, Estudios Científicos, c/ Rosa de Lima, 1, Of. 016, 28230 Las Rozas (Madrid), Spain \\ ${ }^{3} 447$ Spanish odontology, dentistry specialists and maxillo facial surgeons
}

Correspondence:

Medical Director E-C-BIO, S.L,

c/ Rosa de Lima, 1 ,

Edificio ALBA, Office 016,

28230 Las Rozas (Madrid) Spain

bsoler@ecbio.net

Received: 26/03/2010

Accepted: $31 / 05 / 2010$

\begin{abstract}
Bagán JV, Soler-López B, Epico-Study G. Evaluation of the immediate post-operative procedure after dental intervetions. 24 hours follow up study. Epico Study. Med Oral Patol Oral Cir Bucal. 2011 Jul 1;16 (4):e57383.

http://www.medicinaoral.com/medoralfree01/v16i4/medoralv16i4p573.pdf
\end{abstract}

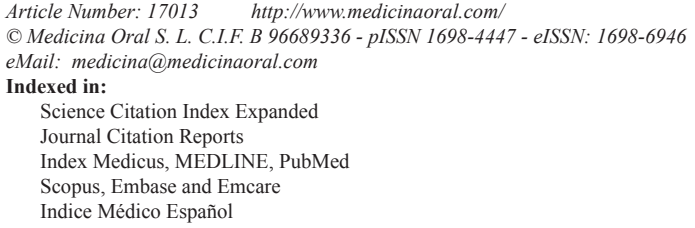

\begin{abstract}
Objectives: The purpose of this study was to make a descriptive approximation of the therapeutic management of the different dental interventions in clinical practice today, and to analyze the degree of consensus among the specialists participating in the study.

Study Design: A total of 447 odontologists, stomatologists or maxillofacial surgeons from 43 Spanish provinces participated in the study. The study sample consisted of patients aged 18 years old or over attending the clinic for a dental intervention. The type of intervention carried out and treatments administered before and after the intervention were recorded. At 24 hours after the intervention, intensity of pain experienced by the patient, rescue therapy administered for pain relief, sleep affectation, the appearance of adverse events or complications of the intervention, and treatment compliance were also recorded.

Results: Data corresponding to 4,194 patients were analyzed, of whom $53.2 \%$ were women $(2,232)$. The mean age was 42.6 years $(95 \%$ CI $42.2-43)$. The most frequent interventions corresponded to: oral surgery (66.4\%), endodontics (17.3\%) and periodontal treatment (4.6\%). A total of $43.2 \%$ of the patients were taking some medication before the intervention: anticoagulants $(2.5 \%)$, antibiotics $(33 \%)$, pain relief and/or anti-inflammatory agents $(23 \%)$ and other drugs (7.6\%); 16.6\% were receiving combined treatment with antibiotics and analgesics and/or anti-inflammatory agents before the intervention. Treatments prescribed to the patient for the 24 hours following the intervention corresponded to only one analgesic and/or anti-inflammatory in 18\%, and a combination of analgesics and/or anti-inflammatories in $77.1 \%$. Antibiotics were prescribed in $39.7 \%$.

Conclusions: Different patterns of therapeutic management by the specialists were noted depending on the type of intervention carried out. The use of analgesics and/or anti-inflammatories depended on the severity of pain expected with each intervention and the administration of antibiotics was related to the prevention of local infections.
\end{abstract}

Key words: Postoperative pain, perioperative treatment, pain prevention, medical management, dental interventions. 


\section{Introduction}

In the numerous interventions carried out in odontology practice, therapeutic and preventive approaches are commonly used without being supported by solid scientific evidence from controlled clinical trials. This occurs in the management of periimplantitis (1), the use of penicillins for prophylaxis of bacterial endocarditis (2), antibiotics to prevent complications after dental implants $(3,4)$, or post-operative pain-relief management. The aim of this study is not to demonstrate that these current interventions are ineffective or unsafe, but rather to show that they are the result of scientific consensus and clinical experience, as opposed to being backed up by well-founded evidence from controlled clinical trials.

Today, there are no published clinical guidelines in our country for post-intervention therapeutic management of odontology patients, and these guidelines are also scarce in other countries (5-7).

The aim of this study is to make a descriptive approximation of the therapeutic management of different dental interventions in Spain, grouped according to the degree of aggression on the gingival environment. We intend for this work to constitute a first step in the development of consensus guidelines for therapeutic management in this specialty, by studying the real situation in clinical practice today and evaluating whether these approaches are supported by well-founded scientific evidence.

\section{Material and Methods}

Study design and ethical considerations

This is an open cohort study with a prospective followup of 24 hours. Patients were included between May and July 2009. The project has received the approval of the Clinical Ethics Committee of the Hospital Clínic i Provincial, Barcelona. All the patients were provided written information on the study and accepted to participate after granting their written consent.

Investigators completed the case report form containing information about the study, by filling in standard forms in electronic format available on the website created especially for the study. Access to the website was restricted and confidential and patients' data were treated anonymously, with patients only being identified by a consecutive numbering system.

\section{Selection of the study objectives}

The main study objective was to assess the different procedures used for the management of patients in the immediate post-operative period, i.e. the first 24 hours following a dental intervention.

Secondary objectives corresponded to studying the pain suffered by patients 24 hours after a dental intervention by applying verbal numeric scales. The need for additional rescue pain-relief to that prescribed by the specialist was assessed, as well as sleep affectation on the night following the dental intervention and the presence of post-operative complications from the dental intervention until the interview 24 hours later.

The verbal numeric scale to assess the intensity of pain after the intervention was a $0-10$ point scale, with 0 indicating "no pain" and 10 indicating the "worst possible pain".

The appearance of adverse events and/or post-operative complications was assessed by asking open questions (i.e. Have you experienced any particular discomfort during the past 24 hours?), after which we explored the appearance of specific symptoms using terms easy for the patient to understand: dry mouth, excess saliva, very sensitive tongue or gums, bleeding gums, nausea, dizziness, drowsiness, headache, vomiting, stomach ache, flatulence, diarrhea.

Patient selection criteria

Patients were selected consecutively by including the first 7 patients attending the dental clinic who fulfilled the inclusion criteria of the study. The data sources used corresponded to the patient's clinical records and the data recorded in the visit when the intervention was carried out. No additional tests were ordered for the study. All patients included were out-patients, of any race and either gender, over 18 years old attending the clinic for a dental intervention. All patients received written information about the study and agreed to participate.

Pregnant women were excluded from the study owing to restrictions in the administration of certain medications during pregnancy.

\section{Treatment}

Since this is a non-interventionist study, the patients selected to participate received treatment and/or medical care for their clinical situation according to the clinical criteria of their specialist.

Before and during the intervention patients were treated according to the standard routine practice of the clinician and site and these treatments were recorded in the case report form for analysis as one of the study objectives. Data was also compiled about the treatments prescribed for the first 24 hours after the intervention, and compliance with these.

\section{Statistical analysis}

E-C-BIO, a clinical research organization, completed the statistical analysis of the study. A descriptive analysis was carried out, showing the distribution of percentages and the $95 \%$ confidence intervals in the qualitative variables, and the usual values (mean, standard deviation, minimum and maximum deviations, 95\% confidence interval) for the quantitative variables. Data were shown in relation to gender, when gender-related differences were evident, and in relation to type of intervention carried out.

Fisher's exact test, and the chi-square test were applied to compare proportions in qualitative variables. 
The Student's t-test for independent samples was used to compare the means in independent groups for the quantitative variables. ANOVA test was applied with Bonferroni or Games Howell corrections for multiple comparisons to study the intensity of pain in relation to different factors (type of intervention, treatment compliance, use of rescue therapy, type of post-intervention treatment, pre and post-operative treatment regimes, sleeping patterns). Multivariate linear regression tests were used to control the effect on intensity of pain, demographic variables, clinical background, therapeutic regimes, therapeutic compliance and the need for rescue therapy. A significance level of 0.05 was considered. The computer software SPSS 14.0 was used for the statistical analysis.

\section{Results}

A total of 447 odontologists, stomatologists or maxillofacial surgeons from 43 Spanish provinces (17 Autonomous Communities) participated in the study, which included 4,194 patients. The inclusion period was from May to July 2009. An average of 9.4 patients $(95 \% \mathrm{CI}$ 9.3 to 9.6 ) were included from each center, with a median of 10 patients per site. A total of 4,221 cases were included, of which the data from 27 patients aged less than 18 years were excluded. The final sample studied corresponded to 4,194 patients who fulfilled all the selection criteria. The percentage of missing data was $0 \%$ in most of the variables provided by the electronic data collection system used for the study. All patients attended and completed the visit 24 hours after the dental intervention ( $0 \%$ drop out rate).

\section{Demographic data and clinical background}

The mean age of patients was 42.6 years $(95 \%$ CI $42.2-$ 43). Table 1 shows a summary of the demographic data of patients included in the study, a description of alcohol and tobacco consumption, and the clinical background in the whole group and according to gender.

A total of $73.8 \%$ of the patients had no clinical background (3,096 patients) and 26.2\% $(1,098)$ had clinical background of some disease, the most frequent being cardiovascular disease $(8.9 \%)$. A higher rate of clinical background was recorded in women than in men $(\mathrm{p}=0.01), 27.8 \%$ (621) of women compared with $24.3 \%$ (477) of men.

\section{Dental intervention}

The type of dental intervention carried out was not specified in 137 patients (3\%) and more than one intervention was carried out simultaneously in 154 patients (3.6\%), which were classified in the multiple intervention group. From table 2 it can be observed that the most common

Table 1. Demographic data and medical background.

\begin{tabular}{|c|c|c|c|c|c|}
\hline & $\mathbf{n}$ & $\begin{array}{l}\text { TOTAL \% (n) } \\
\text { or mean value } \\
(95 \% \mathrm{CI})\end{array}$ & $\begin{array}{l}\text { MEN \% (n) or } \\
\text { mean value } \\
(95 \% \mathrm{CI})\end{array}$ & $\begin{array}{l}\text { WOMEN \% (n) } \\
\text { or mean value } \\
(95 \% \mathrm{CI})\end{array}$ & $\mathbf{P}$ \\
\hline \multicolumn{6}{|c|}{ DEMOGRAPHIC DATA } \\
\hline Gender & 4194 & & $46.8(1962)$ & $53.2(2232)$ & \\
\hline Age & 4194 & $42.6(42.2-43)$ & $42.9(42.2-43.5)$ & $42.4(41.8-43.1)$ & NS \\
\hline \multicolumn{6}{|c|}{ TOBACCO AND ALCOHOL CONSUMPTION } \\
\hline Tobacco & 4194 & $35.7(1496)$ & $41.3(810)$ & $30.7(686)$ & $<0.0001$ \\
\hline Alcohol & 4194 & $15.1(632)$ & $23.7(465)$ & $7.5(167)$ & $<0.0001$ \\
\hline \multicolumn{6}{|l|}{ BODY SYSTEM } \\
\hline ENT & 4194 & $0.6(26)$ & $0.6(11)$ & $0.7(15)$ & NS \\
\hline Respiratory & 4194 & $2.2(93)$ & $2.6(51)$ & $1.9(42)$ & NS \\
\hline Cardiovascular & 4194 & $8.9(375)$ & $10.3(203)$ & $7.7(172)$ & $=0.003$ \\
\hline Gastrointestinal & 4194 & $3.4(142)$ & $3.4(67)$ & $3.4(75)$ & NS \\
\hline Genitourinary & 4194 & $1.5(62)$ & $1.9(37)$ & $1.1(25)$ & $=0.04$ \\
\hline Muscular-skeletal & 4194 & $3.5(145)$ & $2.0(40)$ & $4.7(105)$ & $<0.0001$ \\
\hline Neurological & 4194 & $2.4(101)$ & $1.6(32)$ & $3.1(69)$ & $=0.002$ \\
\hline Endocrinal & 4194 & $5.4(225)$ & $4.4(86)$ & $6.2(139)$ & $=0.008$ \\
\hline Hematological & 4194 & $1.2(49)$ & $0.6(11)$ & $1.7(38)$ & $=0.001$ \\
\hline Dermatological & 4194 & $0.6(27)$ & $0.5(10)$ & $0.8(17)$ & NS \\
\hline Allergies & 4194 & $2.5(104)$ & $1.9(38)$ & $3.0(66)$ & $=0.034$ \\
\hline Neoplasms & 4194 & $0.9(39)$ & $0.8(15)$ & $1.1(24)$ & NS \\
\hline Contagious & 4194 & $0.4(17)$ & $0.7(13)$ & $0.2(4)$ & $=0.014$ \\
\hline
\end{tabular}

95\%CI: 95\% Confidence Interval; p, level of significance; NS: Not significant. Significance levels: CHI2 in categorical variables, Student's t for independent data in quantitative variables (age). 
intervention corresponded to oral surgery (66.4\%), followed by endodontics $(17.3 \%)$, and periodontal treatment (4.6\%).

A greater proportion of women than men were intervened for fixed prosthesis $(2.3 \%$ versus $1.4 \%$ in men, $\mathrm{p}<0.05$ ) and more men than women underwent a multiple intervention $(4.5 \%$ versus $3.2 \%$ in women, $\mathrm{p}<0.05)$.
There were no significant differences between the genders in the remaining interventions.

Medications prescribed before the intervention

At least one of the following medications: anticoagulants, antibiotic or analgesic and/or an anti-inflammatory were administered to $43.2 \%$ of the patients included in the study (1812) $(95 \%$ CI 41.7 to 44.7$)$, in other words,

Table 2. Type of dental interventions carried out and the proportion of patients receiving a single intervention, grouped into seven categories.

\begin{tabular}{|c|c|c|}
\hline INTERVENTION & $\mathbf{N}$ & $\%$ \\
\hline 1. CONSERVATIVE DENTISTRY & & 152 \\
\hline Simple filling (1 surface) & 33 & .8 \\
\hline Compound filling ( 2 surfaces $)$ & 74 & 1.8 \\
\hline Complex reconstruction (major reconstruction/angular reconstruction) & 93 & 2.2 \\
\hline Reconstruction of stump (including posts and/or pins) & 21 & .5 \\
\hline Total interventions & 221 & \\
\hline 2. ENDODONTICS & & 702 \\
\hline Uniradicular endodontics & 174 & 4.1 \\
\hline Biradicular endodontics & 137 & 3.3 \\
\hline Multiradicular endodontics & 386 & 9.2 \\
\hline Re-endodontics & 61 & 1.5 \\
\hline Total interventions & 758 & \\
\hline 3. PERIODONTAL TREATMENT & & 187 \\
\hline Gingivectomy & 73 & 1.7 \\
\hline Periodontal flap surgery & 117 & 2.8 \\
\hline Total interventions & 190 & \\
\hline 4. FIXED PROSTHESES & & 76 \\
\hline Crown & 40 & 1.0 \\
\hline Bridge & 37 & .9 \\
\hline Composite, metal or porcelain incrustation & 2 & .0 \\
\hline Uniradicular die cast stump & 4 & .1 \\
\hline Multiradicular die cast stump & 2 & .0 \\
\hline Extraction of bridge or crown & 12 & .3 \\
\hline Recementing of crowns, veneers, bridge or incrustations & 5 & .1 \\
\hline Total interventions & 102 & \\
\hline 5. ORAL SURGERY & & 2692 \\
\hline Apicoectomy & 66 & 1.6 \\
\hline Simple tooth extraction & 662 & 15.8 \\
\hline Residual root removal & 560 & 13.4 \\
\hline Extraction of submucosal wisdom tooth & 428 & 10.2 \\
\hline Extraction of wisdom tooth or canine in osteotomy or odontosection & 441 & 10.5 \\
\hline Free gingival graft & 13 & .3 \\
\hline Canine ligature. Surgical fenestration of teeth & 9 & .2 \\
\hline Odontogenic cysts & 63 & 1.5 \\
\hline Benign intraoral tumors of soft tissue & 39 & .9 \\
\hline Reimplant for dental avulsion & 1 & .0 \\
\hline Osteointegrated implant & 635 & 15.1 \\
\hline Maxillary sinus elevation & 49 & 1.2 \\
\hline Extirpation of tori or prosthetic exostosis & 18 & .4 \\
\hline Vestibuloplasty & 6 & .1 \\
\hline Total interventions & 2990 & \\
\hline 6. COMPLICATION OF PREVIOUS SURGERY & & 94 \\
\hline Post-operative alveolitis & 96 & 2.3 \\
\hline \multirow[t]{2}{*}{ Other complications } & 60 & 1.4 \\
\hline & 156 & \\
\hline
\end{tabular}


$56.8 \%$ of patients did not receive any treatment before the intervention.

Anticoagulants were being administered before the intervention to a total of $2.5 \%$ of patients (103) $(95 \% \mathrm{CI}$ 2.03 to 2.97). Antibiotics were prescribed before the intervention to $33 \%$ of patients $(1,386)(95 \%$ CI 31.6 to 34.4 ), and the most frequent antibiotics prescribed correspond to amoxicillin or clavulanate-amoxicillin. Analgesics and/or anti-inflammatories were prescribed before the intervention to $23 \%$ of patients (966) $(95 \% \mathrm{CI}$ 21.7 to 24.3$)$.

A total of $16.6 \%(95 \% \mathrm{CI} 15.5$ to 17.7$)$ of patients received simultaneous treatment with antibiotics and/or anti-inflammatories before the intervention.

As regards administration of antioagulants before the intervention, such administration is seen more frequently $(\mathrm{p}<0.05)$ in oral surgery and multiple interventions, while they are less commonly given before conservative surgery, endodontics and periodontal treatment $(\mathrm{p}<0.05)$.
Fig. 1 describes the frequency of administration before the intervention for each group of medications in relation to the intervention carried out. Antibiotics are administered in similar proportions in patients undergoing oral surgery, intervened for a complication from previous surgery, in multiple interventions and in endodontics, in approximately $30 \%$ of cases. However, administration of antibiotics is significantly lower in intervention for fixed prosthesis, conservative odontology and in periodontal treatment $(\mathrm{p}<0.05)$.

Analgesics/anti-inflammatories are administered in similar proportions of around 30\%, in interventions for complications of previous surgery $(42,6 \%)$, endodontics $(32,2 \%)$ and multiple interventions $(27,9 \%)$. In interventions for fixed prosthesis, conservative odontology and periodontal treatment, administration is significantly lower $(\mathrm{p}<0.05)$. These medications are administered to approximately $21 \%$ of cases undergoing oral surgery $(p<0.05)$.

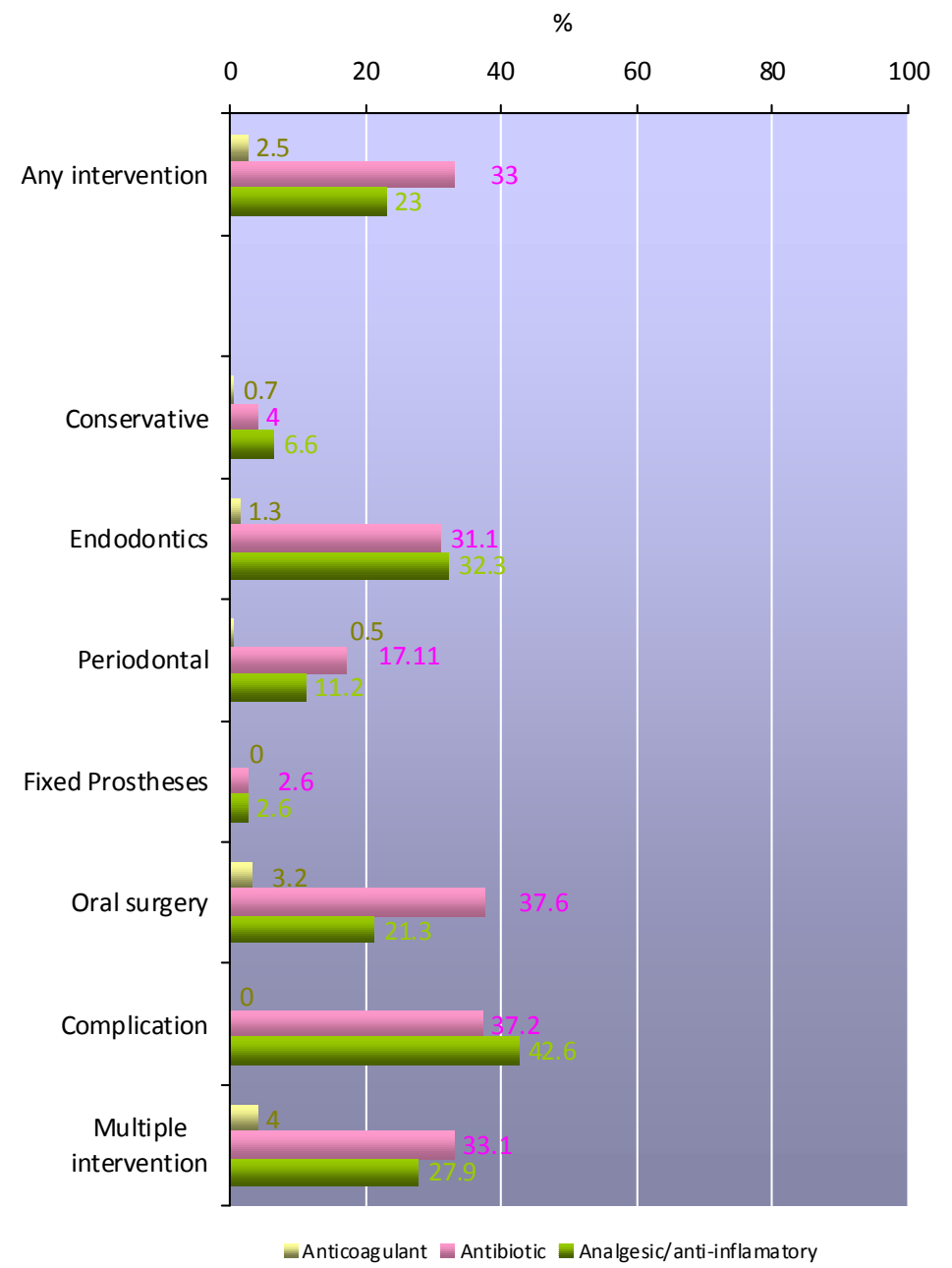

Fig. 1. Medication prescribed BEFORE the dental intervention in relation to type of intervention. 


\section{Medication prescribed after the intervention}

Of the whole group included in the study, $18 \%$ (753) (95\% CI 16.8 to 19.2 ) were prescribed only one analgesic and/or anti-inflammatory over the following 24 hours, $77.1 \%$ (3232) (95\%CI 75.8 to 78.4$)$ were prescribed associations of analgesics and/or anti-inflammatories, and $39.7 \%$ (1663) (95\%CI 38.2 to 41.2$)$ were prescribed an antibiotic.

Fig. 2 shows the frequency of distribution of each group of medications administered after the intervention in relation to the intervention carried out. Antibiotics are prescribed after the intervention to a significantly lower than average proportion of patients in the case of interventions for conservative odontology, endodontics, periodontal treatment, fixed prosthesis and multiple interventions, and in a significantly greater than average proportion in oral surgery and in previous complications $(\mathrm{p}<0.05)$.

A greater proportion of interventions for a previous complication are prescribed analgesic and/or anti-inflammatory associations rather than only one analgesic or anti-inflammatory drug. Prescription of only one an- algesic is significantly greater than average in cases of conservative intervention, endodontics, fixed prosthesis and multiple interventions $(\mathrm{p}<0.05)$.

Analgesic and/or anti-inflammatory associations are prescribed in a greater proportion of interventions for a previous complication $(\mathrm{p}<0.05)$. The prescription of associations is also significantly higher than average $(\mathrm{p}<0.05)$ in periodontal treatment and oral surgery and is significantly lower $(\mathrm{p}<0.05)$ in conservative interventions, endodontics, fixed prosthesis and multiple intervention $(\mathrm{p}<0.05)$.

A total of $95.1 \%$ of patients were prescribed analgesics and/or anti-inflammatories, either as a single product or as an association and $77.1 \%$ of the associated treatments corresponded to a paracetamol/tramadol combination. In $22.3 \%$ of patients, analgesics and/or anti-inflammatories are prescribed before and after the intervention. Prescription of antibiotics and clinical background We have also studied whether the prescription of antibiotics before or after the intervention is related with the presence of any clinical background in the patients. The prescription of antibiotics before the dental inter-

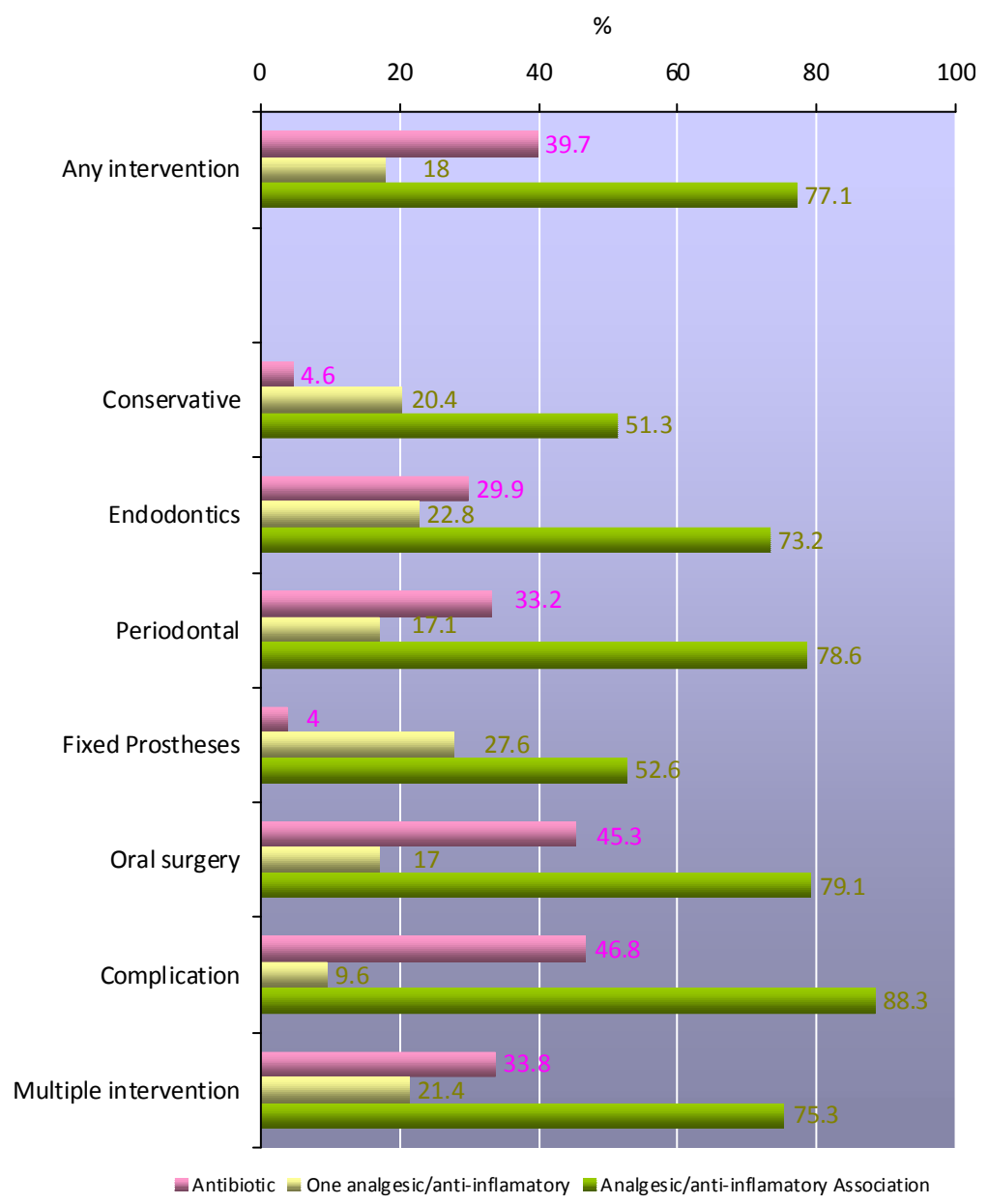

Fig. 2. Medication prescribed AFTER the dental intervention in relation to type of intervention. 
vention is found to be significantly greater $(p=0.009)$ in patients with clinical background $(36.2 \%, 398$ cases of 1,098 patients with a clinical background) than in patients without a clinical background (31.9\%, 988 cases of 3,096 without a clinical background).

Prescription of antibiotics before the intervention is also greater in patients with a background of respiratory disease $(2.9 \%$ vs $1.9 \%)(p=0.039)$, cardiovascular disease $(11.5 \%$ vs $7.7 \%)(p<0.0001)$ or endocrinal conditions $(6.8 \%$ vs $4-7 \%)(p=0.004)$, compared to patients without this background.

In fact, $43 \%$ of patients with respiratory background receive antibiotic treatment before the intervention (40 of 93 patients with a respiratory background).

Of the patients with a cardiovascular background, $42.4 \%$ receive antibiotic treatment before the intervention (159 of 375 patients with a cardiovascular background). Among the patients with an endocrinal background $41.8 \%$ receive antibiotic treatment before the intervention (94 of 225 patients with an endocrinal background).

There is no significant association between the administration of antibiotics after the intervention and the patients' clinical background.

Patients' condition during the 24 hours post-intervention

A total of $84.7 \%$ of patients showed good compliance with treatment with all the drugs prescribed; $68.2 \%$ took the analgesic and/or the anti-inflammatory as indicated and $31.8 \%$ took the medication because they felt pain or discomfort.

A total of $55.5 \%$ of patients reported having experienced pain after the intervention. A significantly higher proportion of women $(58.2 \%)$ than men $(52.5 \%)$ reported suffering pain, $\mathrm{p}<0.0001$.

The mean score on the pain assessment scale for patients feeling pain is 4 points $(95 \% \mathrm{CI}, 3.9$ to 4.1$)$, with a median of 4 points, a minimum value of 0 and a maximum of 10 , this score is on average 0.2 points higher in women than in men $(95 \% \mathrm{CI}, 0.029$ to 0.4$), \mathrm{p}=0.023$.

Fig. 3 shows the mean values for pain in all the patients in relation to the different types of interventions carried out. The mean score is 2.2 points (95\% CI 2.1 to 2.3 ).

Only $5.4 \%$ of patients (228) had to use analgesic and/or anti-inflammatory rescue therapy not prescribed by the clinician. It was noted that a significantly higher proportion of women $(p=0.033)$ than men used rescue therapy not prescribed by the investigator. In fact, $60 \%$ of the patients taking rescue therapy are women and only $39.9 \%$ are men. Among patients who reported suffering from pain, those with the pain of greatest intensity were the ones who required rescue therapy $(p<0.0001)$. Patients using rescue therapy scored an average of 2.04 points more $(95 \% \mathrm{CI} 1.8$ to 2.3$)$ in the pain scale than patients not requiring rescue therapy. It could also be argued that patients using the rescue therapy were the

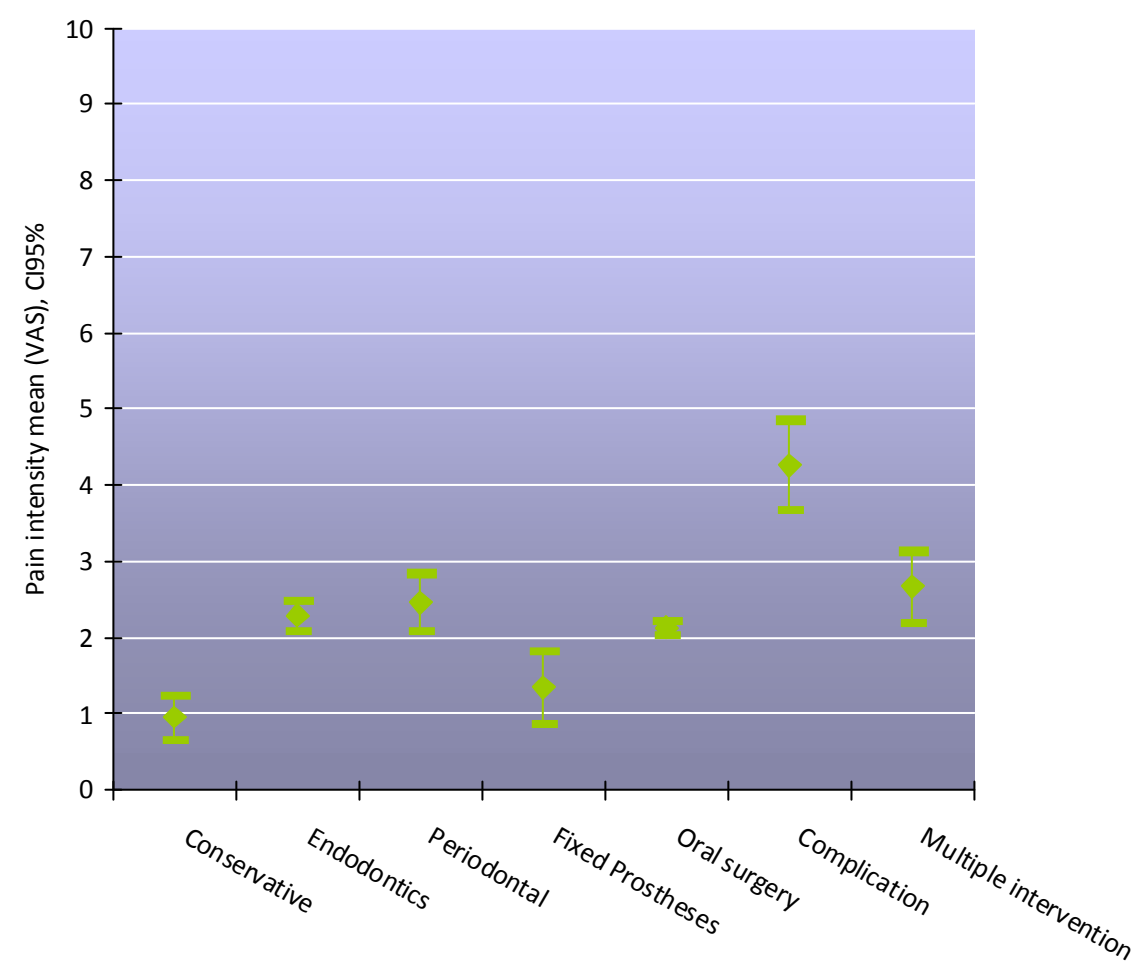

Fig. 3. Assessment of patient's pain on a visual analog scale (VAS) ranging from 0 to 10 points in relation to type of surgical intervention carried out. 
ones who did not take the prescribed medication over the 24 hour period. However, $91 \%$ of the patients requiring rescue therapy had taken the medication prescribed by the odontologist and only $9 \%$ had not taken all the prescribed medication $(\mathrm{p}=0.009)$.

Regarding quality of sleep, $68.7 \%$ of patients reported having slept as well as usual, $22.2 \%$ worse than usual and $9.1 \%$ better than they usually do. Around $81.4 \%$ did not feel restless during the night and $81.9 \%$ of patients did not have to get up during the night. Finally. $67.8 \%$ of patients had not experienced any discomfort in the 24 hours following the intervention, compared to the $32.2 \%(1,351)$ patients who had.

Regarding the adverse effects or complications of the intervention, the frequency of the adverse events was lower than $10 \%$ in each case for all the events recorded. Sorting these in order of decreasing frequency we observe: drowsiness $(384,9.2 \%)$, dry mouth $(308,7.3 \%)$, bleeding gums $(290,6.9 \%)$, headache $(266,6.3 \%)$, dizziness $(257,6.1 \%)$, nausea $(234,5.6 \%)$, hypersalivation (193, 4.6\%), sensitive gums (186, 4.4\%), stomach ache $(172,4.1 \%)$, diarrhea in 87 cases $(2.1 \%)$, vomiting in 67 $(1.6 \%)$ and flatulence in 52 patients $(1.2 \%)$.

Study of factors related with intensity of pain

An analysis was carried out to study a potential association between the intensity of pain and the type of intervention, compliance with the prescribed therapy, use of rescue therapy, type of treatment and sleeping pattern. We could see that patients presented different severities of pain depending on the type of intervention, with the greatest severity being reported after interventions for previous surgical complications $(\mathrm{p}<0.01)$ (Fig. 3).

Scores on the pain scale for multiple interventions are significantly higher than those reported for conservative odontology $(p<0.001)$ and fixed prosthesis $(p=0.04)$ and are significantly lower than those recorded with interventions for a complication of previous surgery $(p<0.0001)$. Periodontal interventions have higher pain score than conservative odontology $(p<0,0001)$ and fixed prosthesis $(p=0.023)$. Endodontic interventions are associated with a greater severity of pain than conservative odontology $(\mathrm{p}<0.0001)$ and fixed prosthesis $(\mathrm{p}=0.04)$.

Regarding the relationship between pain severity and therapeutic compliance, a greater severity of pain is recorded in patients who took all the prescribed drugs $(p<0,001)$ and this pattern is observed in all interventions. Patients who required rescue therapy were also those suffering the greatest pain severity regardless of the type of intervention $(\mathrm{p}<0.001)$. Regarding the medication prescribed, no significant differences were seen between the severity of pain experienced and the prescription of only one analgesic or associations of analgesics and/or anti-inflammatories. However, significant differences were found in relation to whether patients took analgesics and/or anti-inflammatories before and after the intervention or not $(\mathrm{p}=0.001)$.

We noted that, regardless of the type of intervention carried out, on average the patients with the most disturbed sleep were those with the greatest pain severity. Pain severity was greater than in patients who slept the same as usual $(\mathrm{p}<0.0001)$ and those who slept better than usual $(\mathrm{p}<0.0001)$.

We also applied an exploratory multivariate logistic regression in order to control the different variables that could be associated with the presence of pain. Pain severity was also studied by multiple linear regression, which gave significant results in all variables included in the model $(\mathrm{p}<0.0001)$.

We found that older patients are less likely to suffer from pain, OR 0.985 (95\% CI 0.98 to $0.99, \mathrm{p}<0.0001$ ) for each year. On the contrary to what occurs with pain severity, women are less likely to experience pain OR $0,799$ ( $95 \%$ CI 0.7 to $0.913, p=0.001)$; the probability of presenting pain is also lower in patients with a clinical background OR 0.730 (95\%CI 0.618 to $0.863, \mathrm{p}<0.0001)$; and also in patients taking analgesics before the intervention (OR $0.77,95 \% \mathrm{CI} 0.65$ to $0.9, \mathrm{p}=0.001$ ) or with better treatment compliance (OR $0.53,95 \%$ CI 0.45 to $0.64, \mathrm{p}<0.0001)$. Patients requiring rescue therapy are also less likely to present pain (OR $0.07095 \%$ CI 0.04 to $0.13, \mathrm{p}<0.0001)$. Pain was less frequent in patients taking an analgesic and/or anti-inflammatory after the intervention compared with patients not taking any medication (OR $0.695 \% \mathrm{CI} 0.38$ to $0.95, \mathrm{p}=0.28$ ), and was less frequent in those taking an association of analgesics and/or anti-inflammatories compared to those not taking any (OR 0.84 95\% CI 0.71 to $0.997, \mathrm{p}=0.047$ ).

\section{Discussion}

In this analysis of the results of the EPICO study, the sample can be considered to maintain a proportionality in its geographical distribution and also in relation to the frequency of the dental interventions included. It can, therefore, be considered to be representative of the performance of our specialty in clinical practice. The sociodemographic characteristics are similar to those described for the Spanish population in $2008(8,9)$, and the prevalence of disease observed is also comparable to that described in the scientific literature (10).

There are two findings that are especially interesting from the information obtained here. On the one hand, the standard prescription regimes used for the prevention of infectious endocarditis and for the prevention of pain caused by the intervention. The other corresponds to the pain perceived by the patients in relation to the intervention carried out and the treatment received.

The main limitations of this observational study concern the analysis of the intensity of pain perceived by the patients, since the patients taking the prescribed 
drugs will depend on whether these have been prescribed as part of a therapeutic regime or on demand, the product prescribed and the dose, compliance with the prescribed treatment and also the type of intervention carried out. It is, therefore, not possible to draw conclusions about relationships of cause and effect and our analysis is limi-ted to an exploratory approximation using multivariate regression analysis on the presence and intensity of the pain.

In spite of this, we did obtain valuable information on current prescribing patterns, which can open the debate on whether these can be justified by well-founded scientific evidence.

We found that $33 \%$ of patients included in the study receive antibiotic therapy before the intervention and the main indication for this is prophylaxis of infectious endocarditis (IE). However, current recommendations for prophylaxis of IE confine its use to certain heart diseases with a higher risk of IE when a high risk procedure is carried out. In our specialty, this is limited to dental procedures that involve manipulation of the gingival or periapical region of the teeth or perforation of the oral mucosa (Degree of Recommendations Class II a: The weight of evidence supports the use/efficacy, level of evidence C: Consensus of Expert Opinion and/or small studies, routine practice) $(7,11)$.

The prevalence of cardiovascular disease of $8.9 \%$ recorded for the patients included in our study includes any illness classified in this group. However, prophylaxis is currently recommended in a much smaller proportion, and the prescription of antibiotics observed is not justified. Nevertheless, it must be taken into account that the indication of antibiotics prescribed is to prevent local infectious complications and, in this case, the treatment regime is different to that used in the prophylaxis of IE.

Regarding the type of intervention, we have also observed that the use of antibiotics is significantly lower in fixed prosthesis, conservative odontology and periodontal treatment $(\mathrm{p}<0.05)$, and amoxicillin is the preferred antibiotic, in accordance with current recommendations $(7,11)$. It was also found that patients were more likely to be prescribed antibiotics if they had a clinical background ( $p=0.009$ ), and the probability of prescribing antibiotics increased even further in patients with a background of certain types of illnesses, such as cardiovascular, endocrine or respiratory conditions, in this order. Clearly, the rationale for the use of antibiotics must be studied individually for each patient, although it is surprising that almost half of the patients with one of these backgrounds received antibiotic treatment.

Analgesics and/or anti-inflammatories were prescribed before the intervention in around $23 \%$ of cases and there does appear to be scientific evidence from basic research to justify analgesic treatment before an intervention
$(12,13)$. The higher rate of anticoagulants administration in oral surgery and in multiple interventions fig. 1 also seems to be clinically justified.

In the therapeutic action 24-hour post-intervention, $39.7 \%$ of cases were still on antibiotics. Clearly, this prescription pattern cannot be due to prophylaxis for IE but instead must be related to the prevention of local infections, since prophylaxis of IE consists of a single dose administered before the intervention. In order to distinguish this group of patients, we selected the ones who only took antibiotics before the intervention. This corresponded to a total of 810 cases, or $19.3 \%$ of all patients, and is still a higher proportion of patients than those currently recommended for antibiotic prophylaxis. Antibiotics were administered only after the intervention in 1,087 cases $(25.9 \%)$, and were administered before and after the intervention to 576 patients (13.7\%). Prescription of antibiotics after the intervention was more common in oral surgery and in previous complications $(p<0.05)$, which is logical from a clinical perspective.

A total of $95.1 \%$ of patients were prescribed analgesics and/or anti-inflammatories, either as a single product or as an association and $77.1 \%$ of the associated treatments corresponded to a paracetamol/tramadol combination. These drugs are suitable for the treatment of post-surgical and post-traumatic pain, especially when patients are sent home on the day of the operation. The NNT (Number needed to treat), that represent the number of patients needed to treat in order to obtain clinical benefit of one patient, is for paracetamol $(\mathrm{lg})$ around 5 , and that for tramadol $(100 \mathrm{mg})$ is almost as high, and both products are in the middle of the classification table of analgesics for pain relief (14). The drugs were prescribed as part of a therapeutic regime in $68.2 \%$ of cases and compliance with prescribed treatment was very high $(84.7 \%)$.

A total of $55.5 \%$ of patients suffered pain in the 24 hours post-intervention, and a larger proportion of these were women, as was also observed in the scientific literature (14-16). However, this difference is not apparent in the multivariate analysis which did not show a greater proportion of women suffering pain, although the severity of pain suffered by women was found to be greater, but with no clinical relevance. The mean severity of pain of around 2.2 points $(95 \% \mathrm{CI} 2.1$ to 2.3$)$, was similar to that observed in other studies (17), and, as expected, patients presented different patterns of pain severity in relation to the type of intervention, with a greater severity associated with intervention for complications of a previous surgery and multiple interventions. However, our results could possibly be explained by the fact that interventions of longer duration are associated with greater postoperative pain (18).

The fact that patients with better compliance or those requiring rescue therapy presented greater pain intensity does not necessarily imply that the treatment is 
ineffective, since no cause-effect relationship can be deduced in this study. This is similar to when we try to interpret the relationship between the pain and patients not taking medication, taking just one analgesic or anti-inflammatory or taking associated medications. The results are more likely to reflect that patients with less pain take less analgesics or do not take them, rather than that they experience less pain because they do not take the treatment.

In the multivariate logistic analysis we observe that older patients are less likely to suffer pain. Moreover, as we mentioned previously, the presence of pain is less frequent in female patients and in those with a clinical background. Pain is also less frequent in patients who take analgesics before the intervention or in those with better treatment compliance, in agreement with findings reported in the scientific literature (17-19). We also found that pain was less frequent in patients taking an analgesic and/or anti-inflammatory after the intervention compared to patients not taking anything, and was also less frequent in those taking an association of analgesics and/or anti-inflammatories compared to those not taking any medication. It must be admitted that there are numerous other factors that can affect the perception of pain: including environmental, physical and cultural ones, but it was outside the scope of this exploratory analysis to take all these data into consideration.

In summary, the prescription of antibiotics such as we noted in our study is aimed at treating and preventing infectious complications after surgery. The prescription regimes for analgesics and/or anti-inflammatory drugs are clearly determined by the type of intervention carried out, and tend to be used in a significantly greater proportion $(p<0.05)$ in oral surgery and interventions for previous surgical complications. It was found that certain dental interventions resulted in a greater pain for patients and we have obtained a quantitative evaluation of its presence and intensity, and its relationship with other predisposing factors. These values may be used as a reference to make comparisons or predictions in future interventions on our patients, to enable us to achieve a better pain prevention and to make the patient's visit to the odontologist-stomatologist a more satisfactory experience.

To conclude, more work is required in future projects to assess whether our therapeutic protocols improve pain and outcome and reduce complications in our patients. These studies should have a prospective follow up and be homogeneous in relation to the type of intervention and prescribed treatments in order to be able to make appropriate comparisons. Assessment of pain experienced by patients using visual analog scales will give us a quantitative view of the preventive effect of the treatment administered, and the need to be more aggressive in pain-relief treatment.

\section{References with links to Crossref - DOI}

\section{References}

1. Esposito M, Grusovin MG, Kakisis I, Coulthard P, Worthington HV. Interventions for replacing missing teeth: treatment of perimplantitis. Cochrane Database Syst Rev. 2008;2:CD004970.

2. Oliver R, Roberts GJ, Hooper L. Penicillins for the prophylaxis of bacterial endocarditis in dentistry. Cochrane Database Syst Rev. 2004;2:CD003813.

3. Esposito M, Grusovin MG, Talati M, Coulthard P, Oliver R, Worthington HV. Interventions for replacing missing teeth: antibiotics at dental implant placement to prevent complications. Cochrane Database Syst Rev. 2008;3:CD004152.

4. Schwartz AB, Larson EL. Antibiotic prophylaxis and postoperative complications after tooth extraction and implant placement: a review of the literature. J Dent. 2007;35:881-8.

5. Selton-Suty C, Duval X, Brochet E, Doco-Lecompte T, Hoen B, Delahaye E, et al. New French recommendations for the prophylaxis of infectious endocarditis. Arch Mal Coeur Vaiss. 2004;97:626-31.

6. Gould FK, Elliott TS, Foweraker J, Fulford M, Perry JD, Roberts GJ, et al. Guidelines for the prevention of endocarditis: report of the Working Party of the British Society for Antimicrobial Chemotherapy. J Antimicrob Chemother. 2006;57:1035-42.

7. Wilson W, Taubert KA, Gewitz M, Lockhart PB, Baddour LM, Levison M, et al. Prevention of infective endocarditis: guidelines from the American Heart Association: a guideline from the American Heart Association Rheumatic Fever, Endocarditis and Kawasaki Disease Committee, Council on Cardiovascular Disease in the Young, and the Council on Clinical Cardiology, Council on Cardiovascular Surgery and Anesthesia, and the Quality of Care and Outcomes Research Interdisciplinary Working Group. J Am Dent Assoc. 2008;139 Suppl:3S-24S.

8. Instituto Nacional de Estadística, 2008 [homepage on the Internet]. Women and men in Spain 2008, Summary by autonomous communities. Population referred to al 01/01/2008 Royal Decree 2124/2008, of 26 of december. Available in: http://www.ine.es.

9. Instituto Nacional de Estadística [homepage on the Internet]. Madrid: National Health Survey (Encuesta Nacional de Salud); 2006 [13 March 2008]. Available in: http://www.ine.es/jaxi/menu. do? type $=$ pcaxis $\&$ path $=/ \mathrm{t} 15 / \mathrm{p} 419 \&$ file $=$ inebase $\& \mathrm{~L}=0$.

10. Baena Díez JM, Del Val García JL, Tomàs Pelegrina J, Martínez Martínez JL, Martín Peñacoba R, González Tejón I, et al. Cardiovascular disease epidemiology and risk factors in primary care. Rev Esp Cardiol. 2005;58:367-73.

11. Borrás X, Gallego P, Monserrat L. Update on clinical cardiology: aortic diseases, hypertrophic cardiomyopathy, and prophylaxis of infective endocarditis. Rev Esp Cardiol. 2009;62 Suppl 1:28-38.

12. Wall PD. The prevention of postoperative pain. Pain. 1988;33:28990.

13. McQuay HJ, Moore RA. Postoperative analgesia and vomiting, with special reference to day-case surgery: a systematic review. Health Technol Assess. 1998;2:1-236.

14. Karadottir H, Lenoir L, Barbierato B, Bogle M, Riggs M, Sigurdsson $\mathrm{T}$, et al. Pain experienced by patients during periodontal maintenance treatment. J Periodontol. 2002;73:536-42.

15. Hutchinson D, Witt S, Fairpo CG. Pulsed electromagnetic energy therapy in third molar surgery. Oral Surg Oral Med Oral Pathol. 1978;46:748-54.

16. Seymour RA, Blair GS, Wyatt FA. Post-operative dental pain and analgesic efficacy. Part II. Analgesic usage and efficacy after dental surgery. Br J Oral Surg. 1983;21:298-303.

17. Canakçi CF, Canakçi V. Pain experienced by patients undergoing different periodontal therapies. J Am Dent Assoc. 2007;138:156373

18. Curtis JW Jr, McLain JB, Hutchinson RA. The incidence and severity of complications and pain following periodontal surgery. $\mathrm{J}$ Periodontol. 1985;56:597-601.

19. Fardal O, Johannessen AC, Linden GJ. Patient perceptions of periodontal therapy completed in a periodontal practice. J Periodontol. 2002;73:1060-6. 


\section{Acknowledgements-Competing Interests-Funding}

The study has been funded by ZAMBON Spain, who contributed to the investigators selection and review and approved the manuscript.

The principal investigator had full access to all the data in the study and takes responsibility for the integrity of the data and the accuracy of the data analysis. The statistical analysis was completed by Dr. Begoña Soler, Medical Director of E-C-BIO (www.ecbio.net).

We acknowledge the participation of the investigators of the ÉPICO Study Group. 